\title{
Long-term semantic transfer: An overlapping-operations account
}

\author{
ANDREA D. HUGHES and BRUCE W. A. WHITTLESEA \\ Simon Fraser University, Burnaby, British Columbia, Canada
}

\begin{abstract}
Semantic priming is usually a short-lived effect. However, it is also usually investigated using undemanding tasks, such as naming or lexical decision. We instead required subjects to perform category verification. We observed semantically mediated transfer occurring at an average lag of 90 intervening trials. We also observed that this priming was specific to the stimulus properties made salient in prime and probe displays and the particular task demanded in those displays. We interpret the results within an overlapping-operations framework, based on the work of Kolers (e.g., 1976).
\end{abstract}

Semantic priming consists of the facilitation of performing a task, such as naming or lexical decision, when the test stimulus is preceded by a meaningfully or associatively related stimulus. That phenomenon has been extensively explored since it was initially described by Meyer and Schvaneveldt (1971). One of the boundary conditions that has been regularly observed, which has strongly influenced theories about the underlying mechanism, is that it is a short-lived effect, both in absolute time (stimulus onset asynchrony [SOA]) and in terms of lag (number of unrelated items inserted between the related prime and probe). In general, facilitation of the probe is not observed if presentation of the probe is delayed by more than $2 \mathrm{sec}$ (e.g., Neely, 1977, 1991) and can disappear when the SOA is as little as $500 \mathrm{msec}$ (Ratcliff \& McKoon, 1988). Furthermore, although some investigators have observed priming occurring at a lag of 1 (e.g., Joordens \& Besner, 1992; McNamara, 1992), others have found that any amount of lag eliminates the effect (e.g., Masson, 1995).

The dominant explanations of semantic priming have taken this limited duration as a primary characteristic of the effect. The earliest accounts (e.g., J. R. Anderson, 1976, 1983; Collins \& Loftus, 1975; Collins \& Quillian, 1969) were based on the idea of spreading activation. By these accounts, presentation of the prime activates its representation in a semantic network; that activation also spreads along the linkages of the network to all semantically related concepts. In consequence, the representation of a related probe is partially activated prior to its presentation, so that it can be more quickly stimulated above

This research was supported by a grant from the Natural Sciences and Engineering Research Council of Canada. We thank Sachito Kinoshita, Steve Joordens, and James Neely for their thoughtful reviews. We also want to acknowledge Michael Dorken's contributions to the early stages of the development of this project. Copies of the stimuli used in these experiments are available from the authors. Correspondence should be addressed to B. W. A. Whittlesea, Department of Psychology, Simon Fraser University, Burnaby, BC, V5A 1S6 Canada (e-mail: bruce_ whittlesea@sfu.ca). threshold when it is actually shown. By this account, the effect is short-lived because activation dies away quickly in the semantic network. Numerous variations on this type of account have been proposed, including Neely and Keefe's (1989) three-process hybrid theory, which combines automatic spreading activation with the strategic processes of expectancy and semantic matching.

Related to the spreading activation network explanations are the distributed network models (e.g., Masson, 1995; McRae, de Sa, \& Seidenberg, 1997; Plaut \& Booth, 2000). By these accounts, concepts do not have some single representation in a network (a "node"), but instead consist of patterns of activation across a large number of interconnected units in a high-dimensional semantic space. Semantic priming occurs because the pattern of activation across the units is more similar between related primes and probes than it is between unrelated primes and probes, giving the former a head start. By these accounts, presenting another stimulus intervening between the prime and probe interferes with the standing pattern of activation across the network, preventing priming; delay reduces priming for the same reason as in spreading activation networks - the activation pattern decays rapidly over time.

Compound cue models (e.g., Dosher \& Rosedale, 1989; Ratcliff \& McKoon, 1988) offer a quite different explanation. By these accounts, in performing tasks such as lexical decision, the speed of performance will depend on the strength of the familiarity experienced when a cue is presented to memory. Compound cues consist of the composite features of multiple stimuli. A compound cue that is made up of the features of related words presents a superior cue to memory that produces a particularly strong rebound feeling of familiarity, assisting the person in performing lexical decision. Such compounds will often occur when related words are presented back to back; delay of presenting the probe, or inserting another word between the prime and probe, reduces the probability of forming such a compound and thereby eliminates the priming effect.

By all three type of accounts, a short-lived time course is an essential characteristic of semantic priming. In con- 
trast, Whittlesea and Jacoby (1990) presented a "retrieval" account of semantic priming, in which such a time course is not a central issue. ${ }^{1}$ This account was based on Kolers's $(1973,1976)$ idea of "remembering operations," by which any transfer is to be understood as resulting from recapitulating the specific processing operations a person learned to perform on a stimulus within a particular context on a previous occasion: To the extent that the later test involves the same operations as the earlier one, processing will be facilitated.

By the "retrieval" account, the direction of causation underlying semantic priming is from the probe back to the prime, the opposite of that assumed by activation theories. It assumes that processing the prime is a learning experience, like any other stimulus encounter. That experience establishes a resource for performing other, similar, activities on other stimuli that are related on relevant dimensions. That explanation has been applied to long-term priming effects, including repetition priming (Scarborough, Cortese, \& Scarborough, 1977), form-based priming (Ruekl, 1990), and morphological priming (Bentin \& Feldman, 1990). Whittlesea and Jacoby (1990) argued that it can also be applied to semantic priming.

It has long been known that the semantic priming effect is larger when probes are perceptually degraded (e.g., C. A. Becker \& Killion, 1977). This effect is usually interpreted to mean that degradation reveals priming by slowing the perceptual processes leading to identification (e.g., Neely, 1991). Whittlesea and Jacoby (1990) instead proposed that degradation induces priming by forcing the person to rely more heavily on resources made available in other stimulus encounters. According to their retrieval account, the size of the priming effect depends on the need for resources in performing the required task on the probe. If the probe task is easy to accomplish without recruiting resources established on other trials, then there will be little evidence of priming, whereas if the probe task is difficult (e.g., degraded presentation), then performance will rely more heavily on resources made available by other stimulus encounters, causing a larger priming effect. Whittlesea and Jacoby demonstrated that identification of a second presentation of the prime, after the probe had been shown, was facilitated when the probe was degraded. They concluded that information about the prime was more elaborately processed when it was followed by a degraded probe. They argued that, contrary to an activation account, priming is conditional on the need for resources in processing the probe, not an automatic consequence of having encountered the prime.

Whittlesea and Jacoby (1990) did not attempt to extend the time course of semantic priming. However, because their account of priming is based on learning and later retrieval of processing operations, it is open to the possibility that semantic priming can occur over long spans of absolute time and lag of intervening items, just as repetition priming does (e.g., Jacoby \& Dallas, 1981).

S. Becker, Moscovitch, Behrmann, and Joordens (1997) and Joordens and Becker (1997) also proposed that se- mantic priming occurs through a learning process. They proposed a distributed network model similar to those described earlier, but with the critical difference that each occurrence of a stimulus causes permanent changes in the connections of the network. Like Whittlesea and Jacoby's (1990) account, this proposal assumes that processing the prime creates a resource for future processing, a potential that can be realized on later presentation of the probe, rather than automatically preactivating the representation of the probe. It thus admits the possibility of priming of delayed probe presentations. S. Becker et al. further suggested that semantic priming is ordinarily of limited duration over time and lag because the usual test tasks (naming and lexical decision) do not require extensive semantic processing (see Borowsky \& Besner, 1993; Chumbley \& Balota, 1984). To test this idea, S. Becker et al. increased the semantic demand of the task by requiring an animacy decision; they also used multiple primes per probe in order to increase the chance of effective learning. They observed large semantic priming effects at lags up to 8 , and even observed moderate effects at Lags 10 and 21.5. However, in another study, they observed reliable priming at Lags 0, 2, and 8 but not at Lag 4. Joordens and Becker (1997) attempted to increase reliance on semantic processing in lexical decision by presenting very wordlike nonwords (e.g., BRANE). They observed semantic priming effects at Lag 8 in two studies, but failed to observe priming at that lag in two other studies.

Although these results are somewhat inconsistent, they suggest that long-term semantic priming might occur reliably under appropriate prime and probe conditions. One possibility is that S. Becker et al. (1997) and Joordens and Becker (1997) simply did not make the probe task challenging enough. Another is that the operations that the subject learned to perform in encountering the primes were not distinctive enough from the operations that they performed on other trials to show a selective effect of the prior experiences. We began our investigation with that speculation.

\section{EXPERIMENTS 1A AND 1B Long-Term Semantically Mediated Transfer}

Perhaps the most common procedure for studying semantic priming is one in which the subject reads a prime word, then shortly afterward performs lexical decision on a probe item, which is either a word or nonword, and, if a word, either semantically related or unrelated to the prime. Our first study (Experiment 1A) was a simple long-term version of the standard semantic priming experiment. In a prime phase, subjects were asked to name a list of words; in a subsequent probe phase, they were asked to perform lexical decision on words and nonwords. Half of the probe words were semantically related to words shown in the prime phase (e.g., LION-TIGER). A distractor task was inserted between the prime and probe phases. This study was used as a baseline for subsequent experiments. On the basis of much prior research, we did not expect to observe a priming effect in this study. 
In Experiment 1B, like S. Becker et al. (1997), we required the subjects to perform a categorical judgment on primes and probes. However, we modified their procedure so that the decision made on each trial was specific to the prime-probe pair. In this new task, each prime (e.g., EAGLE) was presented along with two category labels (e.g., BIRD and GRASS), one to which the item belonged and one to which it clearly did not belong; the subjects were required to select the correct category as quickly as possible. On the subsequent probe trial, the probe (e.g., HAWK) was presented with the same two categories. On other trials, other primes and probes were shown with different category labels (e.g., COBRA-PYTHON with SNAKE and MACHINE). In this and the following studies, the average delay between the priming event and the probe event was about $20 \mathrm{~min}$ and 90 trials.

\section{Method}

Subjects. Twenty-one Simon Fraser University students participated in Experiments 1A and 1B for course credit.

Procedure. We created a stock of 120 pairs of categorically related words, each pair from a different category (e.g., LION and TIGER [animals], APPLE and ORANGE [fruits], SPRUCE and PINE [trees], and EAGLE and HAWK [birds]). Each category was used only once (i.e., we generated pairs of words from 120 categories). In all of the experiments in this article, half of these pairs (at random for each subject) were assigned to a primed condition, the remainder to be unprimed. If the pair was assigned to the primed condition, the first word of the pair was presented in the first, or prime, phase of the study; the second word of all pairs was presented in the second, or probe, phase. The words within each pair were interchanged for odd-numbered subjects, so that each word served as often as a prime as it did as a probe.

In the prime phase of Experiment 1A, the 60 words selected as primes were shown one at a time, in a freshly randomized order. The subject was to pronounce each word aloud, striking a telegraph key to expose the next word. Between the prime and probe phases of the study, the experimenter chatted with the subjects for $5 \mathrm{~min}$ about unrelated issues, thereby creating an additional separation between encounters with primes and probes. In the probe phase of the study, all 120 words selected as probes were presented, together with 120 nonwords, in a freshly randomized order. Each nonword was generated from one of the natural words by reordering its letters, subject to the restriction that it was easily pronounceable (e.g., PELAP and OGRANE from APPLE and ORANGE). On each test trial, a word or nonword was presented centered on the screen; the subject was asked to perform lexical decision, striking one of two keys to indicate his/her decision.

In this and all subsequent studies, only data from accurately performed probe trials were analyzed. We also eliminated trials on which a reaction latency was more than $2.5 S D$ beyond the mean of its condition, performing this analysis recursively until all latencies were within that limit. In consequence of these elimination proce- dures, on average across subjects and studies, the final analysis was based on about $95 \%$ or more of the trials.

In the prime phase of Experiment 1B, each prime was flanked by two category labels, one appropriate to the prime and the other nonmatching (e.g., ANIMAL-LION-VITAMIN). The subjects were asked to select the category label matching the central word as quickly as possible, using two telegraph keys to indicate left or right. Matching category labels were assigned to the left or right of the prime at random on each trial. As in Experiment 1A, the selection and presentation sequence of items in this phase was freshly randomized for each subject. These procedures were adopted in all later studies as well.

The probe phase was very similar, presenting both primed and unprimed probes along with pairs of category labels. In the case of primed probes, the category labels were identical to those used in training; only the middle word (the probe) was different (e.g., ANIMALTIGER-VITAMIN). However, the category labels were again randomly located to the left and right of the probe, so that the subjects could not make strategic use of a label-side contingency to facilitate processing. This procedure was adopted in all subsequent studies as well. We also examined whether presenting labels on the same side in the prime and probe phase speeded responses relative to changing sides; we observed no evidence of such an effect.

The error rate in this study was only $2 \%$ on average, probably because the correct answer was so clear (e.g., Is EAGLE is a kind of BIRD or MACHINE?). As such, it was too small to support any analysis. Similarly small error rates were observed in the remaining studies.

In this study (unlike later ones) correct and incorrect category labels shown on one probe trial were not presented again on other probe trials. For example, OREGANO and BASIL presented as prime and probe were paired with the category labels SPICE and BONE; neither SPICE nor BONE was shown with any other target word. The nonmatching category labels in this study referred to categories to which no prime or probe used in the study belonged (e.g., kinds of bones were not presented as primes or probes).

\section{Results and Discussion}

In Experiment 1A (lexical decision) there was predictably no evidence of a semantic priming effect $[F(1,20)<1]$ (Table 1). In contrast, in Experiment 1B (category verification), the subjects verified probes prepared by primes about $65 \mathrm{msec}$ faster than unprimed probes $[F(1,20)=$ $\left.15.44, M S_{\mathrm{e}}=4,113.35, p=.001, \eta^{2}=.44\right]$. That is, we observed what appears to be long-term semantically mediated transfer. Like S. Becker et al. (1997), we concluded that that phenomenon occurs when the prime and probe tasks require more extensive semantic processing than is ordinarily invoked by naming or lexical decision. However, the effect in our study was more robust, occurring at an average lag of 20 min and 90 trials. We speculated that requiring the subjects to make different specific decisions on each trial, instead of the same (animacy) decision on each, induced more specifically overlapping operations in

Table 1

Long-Term Lexical Decision and Category Verification (Latency, in Milliseconds)

\begin{tabular}{cllrrr}
\hline Experiment & Prime Phase & Probe Phase & Unprimed & Primed & Difference \\
\hline 1A & Naming & Lexical decision & 902 & 906 & -4 (n.s.) \\
1B & Categorization & Categorization & 1,235 & 1,171 & 64 \\
\hline
\end{tabular}

Note-Experiment 1B: In both phases, mismatched labels referred to categories to which no prime or probe in the study belonged. The same mismatching label was presented with each matching label in the probe phase. 
processing related primes and probes. However, there are a number of possible alternative explanations for this effect. We attempted to eliminate them in the next series of studies.

\section{EXPERIMENTS 2A-2D Control Conditions}

One potential problem with the design of Experiment $1 \mathrm{~B}$ was that the subjects saw the same pair of category labels with the prime and probe (e.g., FRUIT-APPLEMUSIC in the prime phase, FRUIT-ORANGE-MUSIC in the probe phase). Thus, although each label was shown only once in the training, the subjects could have learned to associate a positive response with the matching label and a negative response to the nonmatching label. The resulting facilitation in selecting categories in test might thus have nothing to do with the semantic relationship between the prime and probe per se, but instead result simply from learning to respond or withhold a response to the category labels. We addressed this problem in four stages.

\section{Experiment 2A}

In Experiment 2A, we changed the nonmatching label between prime and probe phases (e.g., FRUIT-APPLEMUSIC in the prime phase, FRUIT-ORANGE-VITAMIN in the probe phase). That at least prevented subjects from using a negative association to reject the mismatching label presented in test. The mismatched labels in both phases referred to categories to which no prime or probe in the study belonged. Otherwise, this study was identical to Experiment $1 \mathrm{~B}$.

\section{Experiment 2B}

However, the subjects could still conceivably learn to make a positive response to the matching label in the prime phase and so be facilitated in responding to it again in the probe phase, without the relationship between the prime and probe having a direct influence. To eliminate this problem, in Experiment 2B, both the matching and nonmatching labels presented on each prime trial would be (or would already have been) presented on some other trial. For example, we might present ANIMAL-LION-FRUIT on one trial, FRUIT-APPLE-BIRD on another, BIRD-EAGLETREE on another, and TREE-SPRUCE-ANIMAL on another. In consequence, every label presented in the prime phase occurred twice, once matching the prime and once not. That condition eliminated the possibility of simple positive and negative response tendencies associated with the category labels. We also changed the pairing of category labels between prime and probe phases, so that if the subject saw ANIMAL-LION-FRUIT in the prime phase, he/she might see instead ANIMAL-LION-BIRD in the probe phase. That eliminated the learning of labels in pairs as a basis for later selection of the correct alternative. We used these procedures for selecting category labels in all subsequent studies.

\section{Experiment 2C}

However, in all of the studies so far discussed, only category labels associated with primed items were presented in the prime phase. (Category labels associated with nonprimed probes could in principle be shown in this phase, but-given that the primes from those sets could not be shown-could only be shown as mismatched labels, introducing other problems.) The facilitation of category verification might thus be due simply to repetition priming in reading the category labels in the probe phase; that is, the subjects might simply identify the names of correct and incorrect categories more quickly when they have seen those names earlier, thus getting a head start on the decision on primed trials. To eliminate this source of influence, in Experiment $2 \mathrm{C}$ we added a preview phase, in which all 120 category labels were exposed, one at a time, in random sequence. The subjects were asked to read each label aloud, in anticipation of an unspecified memory test. This training ensured that the subjects had experienced every category label before test, presented in the same perceptual manifestation (font, size, etc.) in which they would later appear. In all other ways, this study was identical to Experiment 2B. If our priming effect was due to repetition priming of the probes' category labels caused by their presentation in the prime phase, then the effect should at least be substantially reduced in this study relative to Experiment 2B. This preview phase was included in all subsequent experiments.

\section{Experiment 2D}

Finally, we realized that the subjects might not process mismatching labels extensively during the prime phase, and so might not acquire a negative response tendency for mismatching labels. If so, then despite our presentation of each label as both matching and mismatching, the subjects might have learned to respond positively only to category labels presented in the prime phase. It was thus still possible in principle that the locus of the priming effect was speeding of the cognitive or motoric components leading from seeing a label to making a keypress, unmediated by the relationship between the prime and probe. To eliminate this possibility, in Experiment 2D we changed the subjects' task in the priming phase to selecting the category label that did not correspond to the item, thus if anything teaching the subjects to have a selectively negative response tendency to categories shown in the prime phase. At test, as before, the task was to select the category that did match the item. Thus if learning a response to the categories in the prime phase was the source of the priming effect, we could again expect to see a substantial reduction in that effect. In all other ways, this study was identical to Experiment 2C.

\section{Method}

Subjects. Seventeen Simon Fraser University students participated in Experiment 2A, 14 in Experiment 2B, 17 in Experiment 2C, and 23 in Experiment 2D, for course credit.

\section{Results and Discussion}

As shown in Table 2, we observed that verification was faster for primed than unprimed items in all four studies. The size of the effect varied somewhat across the studies 
Table 2

Control Conditions for Category Verification (Latency, in Milliseconds)

\begin{tabular}{ccccccc}
\hline Experiment & Preview & Prime Phase & Probe Phase & Unprimed & Primed & Difference \\
\hline 2A & No & Categorization & Categorization & 1,402 & 1,343 & 59 \\
2B & No & Categorization & Categorization & 1,582 & 1,496 & 86 \\
2C & Yes & Categorization & Categorization & 1,645 & 1,537 & 108 \\
2D & Yes & Categorization & Categorization & 1,652 & 1,593 & 59 \\
\hline
\end{tabular}

Note-Experiment 2A: identical to Experiment 1B, except mismatching labels in the prime phase were replaced by new mismatching labels in the probe phase. Experiment 2B: identical to Experiment $2 \mathrm{~A}$, except that each label presented in the prime phase was presented on two trials, once matching and once nonmatching. Matching labels shown in the prime phase were paired with a different mismatching label in the probe phase. Experiment 2C: identical to Experiment 2B, except for the addition of a preview phase in which all labels to be used in the probe phase were seen and named. Experiment 2D: identical to Experiment $2 \mathrm{C}$, except that in the prime phase, subjects were required to select the nonmatching label.

(being about $60 \mathrm{msec}$ in Experiment $2 \mathrm{~A}[F(1,16)=5.41$, $\left.M S_{\mathrm{e}}=5,489.1, p=.03, \eta^{2}=.25\right], 85 \mathrm{msec}$ in Experiment $2 \mathrm{~B}\left[F(1,13)=6.41, M S_{\mathrm{e}}=7,414.55, p=.03, \eta^{2}=\right.$ $.35], 105 \mathrm{msec}$ in Experiment $2 \mathrm{C}\left[F(1,16)=6.31, M S_{\mathrm{e}}=\right.$ $\left.15,490.35, p=.02, \eta^{2}=.28\right]$, and $60 \mathrm{msec}$ in Experiment $2 \mathrm{D}\left[F(1,22)=4.98, M S_{\mathrm{e}}=8,191.95, p=.04, \eta^{2}=\right.$ $.19]$. However, there was no evidence that any of the control manipulations eliminated or even attenuated the effect we had observed originally in Experiment 1B (about $65 \mathrm{msec}$ ). We concluded that the effect was not due only to the subjects' reactions to category labels in the probe phase, caused either by facilitated encoding of or speeded organization of a response to labels seen in the prime phase, or by differential positive or negative simple or pairwise response tendencies to them. Instead, we concluded that the effect was mediated by the semantic relationship between the prime and the probe. We further suspected that the effect depended on the subjects experiencing the relationship between the category labels and the primes, encoding a representation of that experience and making use of it as a resource in performing the same task on related probes. That speculation was tested in a further pair of studies.

\section{EXPERIMENTS 3A AND 3B Elaboration and Specificity}

In Experiments 1B and 2A-2D, the subjects were required to perform the same category-verification task in both prime and probe phases. From the studies by S. Becker et al. (1997) and Joordens and Becker (1997), we knew that simply performing the same task in both prime and probe phases is insufficient to cause a robust long-term priming effect. However, in principle, the priming we ob- served could have occurred in any of three ways: by establishing a fairly elaborate and distinctive cognitive resource during the priming phase, by presenting a somewhat difficult task in the probe phase (inducing a need for resources in test), or by requiring a somewhat elaborate and distinctive task that was the same in both phases. To test these ideas, in Experiment $3 \mathrm{~A}$ we required the subjects to perform category selection in the prime phase, but lexical decision in the probe phase, using the same nonword stimuli as in Experiment 1A. In Experiment 3B, we instead presented primes without category labels, asking subjects to name them (as in Experiment 1A), but required category verification in the probe phase. Otherwise, these experiments were identical to Experiment $2 \mathrm{C}$.

\section{Method}

Subjects. Twenty Simon Fraser University students participated in Experiments $3 \mathrm{~A}$ and $3 \mathrm{~B}$, for course credit.

\section{Results and Discussion}

As shown in Table 3, requiring categorization of the primes did not induce a long-term priming effect in lexical decision $[F(1,19)<1]$; neither did requiring categorization of the probes following simple naming $[F(1,19)<$ 1]. Comparing the results of these studies with those of Experiments 1 and 2, we concluded that long-term semantic transfer occurs only when the subjects perform elaborate processing on both. We further speculated that it occurs (1) when the test task is reasonably difficult, so that the availability of specifically appropriate processing resources can make a difference in processing efficiency; (2) when those resources have been prepared in the prime phase, by performing either an identical or similar task;

Table 3

Category Verification and Lexical Decision (Latency, in Milliseconds)

\begin{tabular}{ccllcrr}
\hline Experiment & Preview & Prime Phase & Probe Phase & Unprimed & Primed & Difference \\
\hline 3A & Yes & Categorization & Lexical decision & 1,037 & 1,010 & 27 (n.s.) \\
3B & Yes & Naming & Categorization & 1,456 & 1,448 & 8 (n.s.) \\
\hline
\end{tabular}

Note-Experiment 3A: identical to Experiment 2C, except that the probe phase was the same as in Experiment 1A. Experiment 3B: identical to Experiment 2C, except that the prime phase was the same as in Experiment 1A. 
and (3) when the resources needed to perform the probe task differ from trial to trial. We also speculated that a "specifically appropriate cognitive resource" consists of an earlier experience involving operations that overlap those required to perform the probe task (see Kolers, 1973, 1976). We attempted to test the overlapping-operations hypothesis in the next pair of studies.

\section{EXPERIMENTS 4A AND 4B Breadth of Transfer}

In the previous studies, the subjects were asked to verify an abstract proposition involving membership in a class. Our next question was whether such priming occurs only at that abstract level, or instead whether facilitation would occur more generally, whenever the skill acquired in processing the prime was appropriate for dealing with the task required in processing a semantically related probe. In Experiment 4A, we replaced all of the category labels with another exemplar from the same class. Thus, for example, a subject might see PEAR-APPLE-CROW in the prime phase and PEAR-ORANGE-COBRA in the test. The subjects were asked to select on each trial the flanker word belonging to the same category as the middle word. In Experiment $4 \mathrm{~B}$, we replaced the exemplar labels with the name of a feature (an action, abstract property, or physical part) associated with the prime and probe. Thus, for example, a subject might see PEEL-APPLE-FEATHER in the prime phase and PEEL-ORANGE-HISS in the test. The subjects were asked to select on each trial the flanker word representing a feature associated with the middle word. In all other ways, these studies were identical to Experiment $2 \mathrm{C}$.

\section{Method}

Subjects. Eighteen Simon Fraser University students participated in Experiment 4A and 15 in Experiment 4B, for course credit.

\section{Results and Discussion}

As shown in Table 4, we observed speeded verification of both related exemplars and features, of about $100 \mathrm{msec}$ $\left[F(1,17)=5.59, M S_{\mathrm{e}}=16,429.26, p=.03, \eta^{2}=.25\right]$ and $150 \mathrm{msec}\left[F(1,14)=7.84, M S_{\mathrm{e}}=16,657.86, p=.02\right.$, $\left.\eta^{2}=.24\right]$, respectively. We concluded that the long-term semantic transfer effect can be mediated broadly by preparing the subjects to process relationships, at a variety of levels of abstractness, between a probe and an associated concept.
However, it was not clear from those studies whether the observed transfer was specific to the particular relationship that we had primed. That is, causing the subject to think about the relationship between a prime and some particular level of associate (class, exemplar, or feature) might facilitate processing of the probe task only when that task involved the same level of relationship. Alternatively, the priming might be less specific. For example, asking the subject about any level of associate of the prime (its class, another exemplar, or a specific feature) might automatically activate semantic information at other levels, perhaps mediated through activation of some basic level of categorization (see Rosch, 1978). If that occurred, then we could expect to observe fairly broad transfer, such that presenting a prime task at one level of abstraction would facilitate performance on a probe task involving a different level of abstraction.

\section{EXPERIMENTS 5A AND 5B Specificity of Transfer}

To test this issue of specificity, in Experiment 5A we presented category labels as flankers in the prime phase, but features in the probe phase. Thus a subject might see FRUIT-APPLE-BIRD in the prime phase and PEEL-ORANGEHISS in the test. In Experiment 5B we reversed this procedure, presenting features as flankers in the prime phase, but categories in the probe phase. In all other ways, these studies were identical to Experiments 4A and 4B.

\section{Method}

Subjects. Nineteen Simon Fraser University students participated in Experiment 5A and 13 in Experiment 5B, for course credit.

\section{Results and Discussion}

As shown in Table 5, we observed no reliable evidence of facilitated verification of features when categories were shown with primes $(F<1)$, or of facilitated verification of categories when features were shown with primes $(F<1)$. We concluded that the processing operations involved in performing each of these activities were different enough that the subjects could not make effective use of their earlier experiences as resources in performing the later task. We further concluded that long-term semantic priming does not occur through broad activation of conceptual associates of primed items. Thinking about the category to which an exemplar belongs does not activate information about the features of the exemplar, nor does thinking

Table 4

Associate and Feature Verification (Latency, in Milliseconds)

\begin{tabular}{ccccccc}
\hline Experiment & Preview & Prime Phase & Probe Phase & Unprimed & Primed & Difference \\
\hline 4A & Yes & Exemplar & Exemplar & 1,637 & 1,536 & 101 \\
4B & Yes & Feature & Feature & 1,924 & 1,777 & 148 \\
\hline
\end{tabular}

Note-These studies were identical to Experiment 2C, except that related versus unrelated exemplars were shown as flankers in Experiment $4 \mathrm{~A}$ and related versus unrelated features were shown in Experiment 4B. 
Table 5

Feature and Category Verification (Latency, in Milliseconds)

\begin{tabular}{ccccccc}
\hline Experiment & Preview & Prime Phase & Probe Phase & Unprimed & Primed & Difference \\
\hline 5A & Yes & Category & Feature & 1,797 & 1,775 & 22 (n.s.) \\
5B & Yes & Feature & Category & 1,493 & 1,478 & 15 (n.s.) \\
\hline
\end{tabular}

Note-These experiments were identical to Experiment 2C, except for the presentation of related versus unrelated features in the probe phase of Experiment $5 \mathrm{~A}$ and in the prime phase of Experiment 5B.

about the features of the exemplar prepare the person to answer questions about the category to which it belongs.

\section{GENERAL DISCUSSION}

\section{Summary}

Experiment 1 A predictably demonstrated that no priming occurs in a lexical decision task when primes and probes are widely separated in time and trials. In contrast, Experiment 1B demonstrated a robust long-term priming effect, in which novel probe words that were semantically related to words in the priming phase were categorized more swiftly than those that were not. Four control conditions in Experiment 2 eliminated a variety of sources of that effect, including acquisition of simple or pairwise positive and/or negative response tendencies to matching versus nonmatching category labels, differential exposure to category labels of primed and unprimed items prior to the probe phase, and differential degrees of processing matching versus nonmatching labels in the prime phase. We concluded that the priming effect was not the result of learning to respond to the labels themselves. Instead, it was a transfer effect, mediated by the semantic relatedness of primes and probes. That transfer effect occurred because the subjects experienced the relationship between primes and their categories, formed an enduring representation of that experience, and used that representation as a resource in performing a similar decision on related probes.

Experiment 3 demonstrated that category verification is not by itself sufficient to cause the priming effect, whether that task is performed on primes in advance of a lexical decision test on probes or whether it is required on probes after naming primes. That suggested that the long-term effect occurs only when extensive semantic processing is required in each phase. It might also imply that the effect depends on the subject performing the same task in both phases. That idea is consistent with the results of Experiment 4 , in which the effect was again observed when the task was the same in both phases (exemplar-exemplar and feature-feature). However, we do not draw such a strong conclusion. Kolers's $(1973,1976)$ overlapping-operations framework predicts priming even when the tasks in the two phases are nonidentical, as long as procedures necessary to perform the probe task are prepared by elements of whatever the subject does in interacting with primes. Requiring the same verification task with the same flanker words, albeit with different target words, is an extreme case of overlapping operations, but is unlikely to be necessary to observe some priming. That issue remains to be explored.
Experiment 4 also demonstrated that the effect is not restricted to category-level processing, but is just as robust in comparing category members to other exemplars or verifying their features. That suggests that the effect occurs whenever some particular aspect of the meaning of a word that is interrogated in a probe task is also involved in the processing of a related word in the prime task.

Experiment 5 placed another boundary on the effect: When the task changed from verification of category to verification of feature between phases, or vice versa, the priming effect was numerically present, but much smaller and no longer reliable. We interpret that within the overlapping-operations framework to mean that the cognitive procedures conducted to perform one task were insufficiently similar to those required in performing the other to be a useful resource for making the decision. Perhaps more important, that specificity denies that the priming observed in the earlier studies was mediated by activation of an abstract, context-free, "core meaning" of those words.

Comparing our results with those of S. Becker et al. (1997) suggests one further condition for observing robust long-term semantically mediated priming: that the processing on each trial be different (semantically distinctive) from that on other trials. We have not tested the importance of that factor directly within these studies. Nor do we have evidence about whether such distinctiveness is important exclusively on prime trials, probe trials, or both. We suspect that it may have several effects. First, in tailoring the matching labels (categories, exemplars, and features) specifically to each prime-probe pair, we probably allowed the subjects to perform more extensive semantic processing in both phases than was possible in answering the "animacy" question used by S. Becker et al. Second, however, the trial-by-trial differences in the priming phase may have created more distinctive representations of experience that could be cued more selectively on probe trials. Similarly, the trial-by-trial differences in the probe phase may have caused them to be more effective cues for selective recapitulation of earlier processing. Third, more distinctive processing may have led the subjects to a greater feeling of definiteness of answer, allowing them to respond more confidently and so more quickly on primed trials.

\section{Long-Term Semantically Mediated Transfer: Semantic or Repetition Priming?}

We clearly observed a long-term transfer effect, occurring within the context of conceptual decisions and mediated by the semantic relation between primes and probes. The question to be answered now is what this effect has to 
do with the classic phenomena of semantic and repetition priming.

Woltz (1996) distinguished between two ways in which earlier experiences could, in principle, enhance later conceptual processing in the long term: by increasing the $a c$ cessibility of the meaning of a word, or by enhancing the process of comparing the meaning of that word to some other word or concept. The difference, operationally, is that the former would enhance the fluency of performing any conceptual task on that word, the latter only the efficiency of performing tasks involving similar decisions. It seems clear from Experiments 3, 4, and 5 that the priming observed here depends on reenacting specific cognitive operations performed earlier. That is, the experience of classifying the prime does not simply make the abstract meaning of the probe more accessible, but instead facilitates specific kinds of judgements about the probe that were also made about its prime. One might therefore be tempted to think of this phenomenon as a kind of repetition priming. The problem with that idea is that, unlike the standard repetition priming paradigm, target words presented in the probe phases in our experiments were not repetitions, but instead words semantically related to words in the prime phases. To refer to these results as "repetition priming" forces one to redefine that phenomenon as "facilitation resulting from having had the experience of performing some operations on some stimulus that can be used as a resource in performing some task on some other stimulus, including but not restricted to exact repetition." That is, the priming that we observed is an example of repetition priming only if that effect is redefined as "overlapping operations."

Is the phenomenon that we observed also an example of long-term semantic priming? Proponents of the activation account of semantic priming (described earlier) appear to have adopted an assumption similar to Woltz's (1996) other alternative, that the prime increases the sheer accessibility of the meaning of the probe. Given such an assumption, our effect is not semantic priming. However, we are not aware of any direct evidence in favor of that assumption, even in short-term tests. The most direct evidence of that idea would be a convincing demonstration of heightened but context-free and task-independent efficiency in semantic processing. Instead, the literature provides a complex and conditional picture of the relationship between processing of a prime and probe, even in the short term. First, both associative and categorical relationships between the prime and probe have been demonstrated to facilitate performance (e.g., Lucas, 2000). Second, the term semantic priming is applied to facilitation effects found both in naming of probes and lexical decision, although those tasks can involve the use of different resources (Seidenberg, Waters, Sanders, \& Langer, 1984). Third, although it was initially conceived to be a purely automatic effect (e.g., Meyer \& Schvaneveldt, 1971), the semantic priming effect has also been shown to be influenced by strategic processes, including both expectation and semantic matching, at least under SOAs longer than about 500 msec (C. A.Becker, 1980; Neely, 1977). Further, although some researchers have reported evidence of priming occurring when the prime is processed subliminally, suggesting that no special processing of the prime is required to produce it (e.g., Balota, 1983), others have demonstrated that priming is attenuated when the subject is led to process the prime in a superficial way (e.g., Stolz $\&$ Besner, 1999), suggesting that the effect is mediated by the specific processes applied to the prime. Finally, the term semantic priming is also applied to cases in which the association between prime and probe is asymmetric, although forward versus backward associations may influence processing in quite different ways (see McNamara $\&$ Holbrook, 2003, for a review of this issue).

In the face of such variability of experimental procedures and outcomes, it is hard to say whether or not a given observation represents an example of the same general phenomenon, and must be taken into account in coming to a general explanation of it. However, we take it that the defining characteristic of semantic priming is that the facilitation of performance on a stimulus is mediated by its semantic or associative relationship with a prior stimulus, rather than by some other aspect of the situation. In our experiments, we have attempted to eliminate every alternative form of mediation. By that standard, we suggest that the long-term transfer effect observed in these studies is part of the semantic priming phenomenon: Any complete account of that effect must be able explain these results as well.

It is, of course, possible to accept our results as an example of semantically mediated transfer, but to argue that such transfer occurs by different principles in the long and short terms. That argument might rescue the spreadingactivation account of semantic priming (although that account still has difficulties in explaining effects such as those observed by Whittlesea \& Jacoby, 1990). However, the overlapping-operations hypothesis works as well to explain observations of short-term priming as it does in the long term. On grounds of generality, it is the preferable hypothesis.

\section{How Distinct Are Semantic and Repetition Priming?}

There are also a number of other demonstrations of long-term conceptual priming that should be taken into account in producing a general theory of conceptual transfer. For example, Vriezen, Moscovitch, and Bellos (1995) observed that subjects making judgments of either big/ small or natural/artificial experienced priming when words were repeated in test and the subjects made the same decision. However, priming was not observed when the original presentation required a nonsemantic task such as lexical decision or naming; more importantly, priming was not observed when the original presentation involved the alternate semantic decision. Similarly, Woltz (1996) demonstrated that deciding that two synonyms (e.g., DAMP and MOIST) are related is enhanced by prior experience of the same pair in the same decision. However, priming did 
not occur when the same two words were presented earlier on different trials (e.g., presenting DAMP-DAMP and MOIST-MOIST on separate trials), indicating that the priming was specific to reenacting operations performed on earlier trials. The effects in both of those studies really are cases of "repetition priming" (the same stimulus as well as the same task being presented in both phases of the study); but in both cases, the priming was mediated by an experience of thinking about the stimulus in a particular way, not by activation of its general, abstract meaning or through its perceptual characteristics.

Moreover, Blaxton (1989) observed conceptual priming occurring in the absence of actual repetition. She demonstrated that answering general knowledge questions (e.g., What is the fastest animal on earth?) is facilitated after a long delay by earlier generation of the answer from semantic cues. In that case, the later test was conceptual and involved the same word as the earlier experience, but the test was not identical to the earlier experience. However, Blaxton also observed that the priming was specific to the relationship between the demands of earlier and later processing: Priming did not occur when the same answer had been generated from word fragments. McDermott (1997) also investigated conceptual priming in the absence of actual repetition. She observed that presentation of a list of 15 semantic associates (e.g., NIGHT, BED, DREAM, etc.) caused priming for the semantic prototypes of those lists (SLEEP, in the example) in a delayed test emphasizing conceptual properties (word association). In that case, transfer occurred to a word that had not been exposed earlier, but that was highly semantically related to a list of words that had been. However, the occurrence of priming was specific to the demands of the test: It did not occur in a test emphasizing perceptual processing (word stem and fragment completion).

These sets of results, like ours, do not fit tidily into a dichotomy of semantic versus repetition priming: All involve long-term priming in some conceptual task rather than a perceptual or identification task, but all involve repetition or at least carryover of some specific way of dealing with stimuli in training and test. In breaking down the paradigmatic differences between repetition priming and semantic priming, they further suggest that those effects do not result from a dichotomy of principles, each effect being controlled by a different function or structure of memory. Instead, they suggest that the classic findings of short-lived, conceptually mediated semantic priming and enduring, perceptually mediated repetition priming may be just local cases within a much larger field of effect governed by Kolers's $(1973,1976)$ unifying principle of overlapping operations. The parameters of that field appear to include not just the conceptual and perceptual relationship between stimuli presented on earlier and later occasions, but critically also the specific demands of the task that the subject is attempting to perform on each of those occasions, the operations necessary to satisfy those demands, and the resources made available to perform those operations by previous experiences of processing other (struc- turally or semantically similar or dissimilar) stimuli for the same or other purposes.

\section{Implications}

More broadly, the interpretation of the present effect is part of a larger debate about how to conceive of the fundamental operation of mind. The opposed alternatives are, roughly, the "structural" and "processing" accounts. Structural accounts place fundamental stress on the idea that mind has an architecture, and that this architecture is responsible for the variety of performance that can be observed. Investigators making this assumption make use of concepts such as activation, inhibition and decay, network organization, and strength (rather than quality) of representation. These accounts propose separate memory systems for remembering versus nonremembering activities, such as semantic versus episodic (e.g., Tulving, 1983, 1995), procedural versus declarative (Cohen \& Squire, 1980; Knowlton \& Squire, 1995), or implicit versus explicit (Gabrieli, 1998; Graf \& Schacter, 1987). Whereas remembering is thought to involve the encoding and retrieval of particular, context-defined elements of particular experiences, and to be controlled by principles such as encoding variability and specificity (transfer-appropriate processing), perceptual and conceptual learning and performance are thought to occur primarily though a process of chronic, automatic, abstraction, resulting in a stable, monolithic representation. By these accounts, many of the phenomena of perceptual and conceptual performance are assumed to be transitory events in an otherwise stable system. A number of interference phenomena have been interpreted in that way, including negative priming (e.g., Tipper, 1985; Tipper, Meegan, \& Howard, 2002), the Stroop effect (Logan, 1980), attentional blink (Chun \& Potter, 1995), repetition blindness (Kanwisher, 1987; Morris \& Harris, 2002; Neill, Neely, Hutchison, Kahan, \& VerWys, 2002), the inhibition of return effect (Klein, 1988), and so on. Each of those effects has been explained through inhibition automatically spreading through some type of network, momentarily perturbing the state of some parts of the network and preventing it from performing some behavior that would normally be expected, but leaving behind little residual effect. ${ }^{2}$

In contrast, processing accounts tend to assume that all cognitive and perceptual events occur through the same set of principles. Every stimulus encounter is a learning event, which can have consequences for later processing; each experience is preserved, rather than being amalgamated into an abstract, monolithic representation. However, the central principle of these accounts is that each processing event consists of a particular combination of task, context, stimulus properties, and cued memory traces (see Whittlesea \& Leboe, 2000). In consequence, transfer effects, even in the short term, are expected to show effects of the specific demands and affordances of particular processing events and also the specific relationships between those events and others that the person has experienced. Processing accounts have been offered for many 
of the same phenomena, including negative priming (e.g., Milliken, Joordens, Merikle, \& Seiffert, 1998), the Stroop effect (MacLeod, 1998), repetition blindness (Masson, Caldwell, \& Whittlesea, 2000), and the inhibition of return effect (Pratt, Spalek, \& Bradshaw, 1999).

No general conclusion can yet be drawn about the best way to conceptualize the fundamental organization of memory. However, in showing that semantic priming can be a long-term effect, but more importantly in showing that such long-term effects are specific to the particular stimulus properties the person encounters and the particular operations the person conducts, the present data add one more piece of evidence in favor of a general processing account of mind.

\section{REFERENCES}

ANDERSON, J. R. (1976). Language, memory and thought. Hillsdale, NJ: Erlbaum.

Anderson, J. R. (1983). The architecture of cognition. Cambridge, MA: Harvard University Press.

Anderson, M. M. C., BJork, R. A., \& Bjork, E. L. (1994). Remembering can cause forgetting: Retrieval dynamics in long-term memory. Journal of Experimental Psychology: Learning, Memory, \& Cognition, 20, 1063-1087.

Balota, D. A. (1983). Automatic semantic activation and episodic memory encoding. Journal of Verbal Learning \& Verbal Behavior, 22, 88-104.

BeCKer, C. A. (1980). Semantic context effects in visual word recognition: An analysis of semantic strategies. Memory \& Cognition, 8, $493-$ 512.

Becker, C. A., \& Killion, T. H. (1977). Interaction of visual and cognitive effects in word recognition. Journal of Experimental Psychology: Human Perception \& Performance, 3, 389-401.

Becker, S., Moscovitch, M., Behrmann, M., \& Joordens, S. (1997) Long-term semantic priming: A computational account and empirical evidence. Journal of Experimental Psychology: Learning, Memory, \& Cognition, 23, 1059-1082.

Bentin, S., \& Feldman, L. (1990). The contribution of morphologic and semantic relatedness to repetition priming at short and long lags: Evidence from Hebrew. Quarterly Journal of Experimental Psychology, 42A, 693-711.

BJORK, R. A. (1989). Retrieval inhibition as an adaptive mechanism in human memory. In H. L. Roediger III \& F. I. M. Craik (Eds.), Varieties of memory and consciousness: Essays in honour of Endel Tulving (pp. 309-330). Hillsdale, NJ: Erlbaum.

BlaXton, T. A. (1989). Investigating dissociations among memory measures: Support for a transfer-appropriate processing framework. Journal of Experimental Psychology: Learning, Memory, \& Cognition, 15, 657-668.

Borowsky, R., \& BESNER, D. (1993). Visual word recognition: A multistage activation model. Journal of Experimental Psychology: Learning, Memory, \& Cognition, 19, 813-840.

Chumbley, J. I., \& Balota, D. A. (1984). A word's meaning affects the decision in lexical decision. Memory \& Cognition, 12, 590-606.

Chun, M. M., \& Potter, M. C. (1995). A two-stage model for multiple target detection in rapid serial visual presentation. Journal of Experimental Psychology: Human Perception \& Performance, 21, 109-127.

Cohen, N. J., \& SQuire, L. R. (1980). Preserved learning and retention of pattern-analyzing skill in amnesia: Dissociation of knowing what and knowing how. Science, 210, 207-210.

Collins, A. M., \& Loftus, E. F. (1975). A spreading-activation theory of semantic priming. Psychological Review, 82, 407-428.

Collins, A. M., \& Quillian, M. R. (1969). Retrieval time from semantic memory. Journal of Verbal Learning \& Verbal Behavior, 8, 240-247.

Dosher, B. A., \& Rosedale, G. (1989). Integrated retrieval cues as a mechanism for priming in retrieval from memory. Journal of Experimental Psychology: General, 118, 191-211.
Gabrieli, J. D. E. (1998). Cognitive neuroscience of human memory. Annual Review of Psychology, 49, 87-115.

Graf, P., \& Schacter, D. L. (1987). Selective effects of interference on implicit and explicit memory. Journal of Experimental Psychology: Learning, Memory, \& Cognition, 13, 45-53.

JACoвy, L. L., \& Dallas, M. (1981). On the relationship between autobiographical memory and perceptual learning. Journal of Experimental Psychology: General, 110, 306-340.

JoordEns, S., \& BECKER, S. (1997). The long and short of semantic priming effects in lexical decision. Journal of Experimental Psychology: Learning, Memory, \& Cognition, 23, 1083-1105.

JooRdEns, S., \& BESNER, D. (1992). Priming effects that span an intervening unrelated word: Implications for models of memory representation and retrieval. Journal of Experimental Psychology: Learning, Memory, \& Cognition, 18, 483-491.

KANWISHER, N. G. (1987). Repetition blindness: Type recognition without token individuation. Cognition, 27, 117-143.

KLEIN, R. (1988). Inhibitory tagging system facilitates visual search. Nature, 334, 430-431.

Knowlton, B. J., \& SQuire, L. R. (1995). Remembering and knowing: Two different expressions of declarative memory. Journal of Experimental Psychology: Human Perception \& Performance, 21, 699-710.

Kolers, P. A. (1973). Remembering operations. Memory \& Cognition, 1, 347-355.

Kolers, P. A. (1976). Reading a year later. Journal of Experimental Psychology: Learning, Memory, \& Cognition, 2, 554-565.

LogAN, G. D. (1980). Attention and automaticity in Stroop and priming tasks: Theory and data. Cognitive Psychology, 12, 523-553.

LuCAS, M. (2000). Semantic priming without association: A meta-analytic review. Psychonomic Bulletin \& Review, 7, 618-630.

MACLEOD, C. M. (1998). Training on integrated versus Stroop tasks: The progression of interference and facilitation. Memory \& Cognition, 26, 201-211.

Masson, M. E. J. (1995). A distributed memory model of semantic priming. Journal of Experimental Psychology: Learning, Memory, \& Cognition, 21, 3-23.

Masson, M. E. J., Caldwell, J. I., \& Whittlesea, B. W. A. (2000). When lust is lost: Orthographic similarity effects in the encoding and reconstruction of rapidly presented word lists. Journal of Experimental Psychology: Learning, Memory, \& Cognition, 26, 1005-1022.

McDermott, K. B. (1997). Priming on perceptual implicit memory tests can be achieved through presentation of associates. Psychonomic Bulletin \& Review, 4, 582-586.

MCNamara, T. P. (1992). Theories of priming: I. Associative distance and lag. Journal of Experimental Psychology: Learning, Memory, \& Cognition, 18, 1173-1190.

McNamara, T. P., \& Holbrook, J. B. (2003). Semantic memory and priming. In I. B. Weiner (Series Ed.) \& A. F. Healy \& R. W. Proctor (Vol. Eds.), Handbook of psychology: Vol. 4. Experimental psychology (pp. 447-474). New York: Wiley.

McRae, K., De SA, V., \& Seidenberg, M. S. (1997). On the nature and scope of featural representations in word meaning. Journal of Experimental Psychology: General, 126, 99-130.

Meyer, D. E., \& Schvaneveldt, R. W. (1971). Facilitation in recognizing pairs of words: Evidence of a dependence between retrieval operations. Journal of Experimental Psychology, 90, 227-234.

Milliken, B., Joordens, S., Merikle,P. M., \& Seiffert, A. E. (1998). Selective attention: A re-evaluation of the implications of negative priming. Psychological Review, 105, 203-229.

Morris, A. L., \& HARrIS, C. L. (2002). Sentence context, word recognition, and repetition blindness. Journal of Experimental Psychology: Learning, Memory, \& Cognition, 28, 962-982.

NeELY, J. H. (1977). Semantic priming and retrieval from lexical memory: Roles of inhibitionless spreading activation and limited capacity attention. Journal of Experimental Psychology: General, 106, 226-254.

Neely, J. H. (1991). Semantic priming effects in visual word recognition: A selective review of current findings and theories. In D. Besner \& G. W. Humphreys (Eds.), Basic processes in reading: Visual word recognition (pp. 264-336). Hillsdale, NJ: Erlbaum.

Neely, J. H., \& KeEFe, D. E. (1989). Semantic context effects on visual word processing: A hybrid prospective/retrospective processing the- 
ory. In G. H. Bower (Ed.), The psychology of learning andmotivation (Vol. 24, pp. 207-248). New York: Academic Press.

Neill, W. T., Neely, J. H., Hutchison, K. A., Kahan, T. A., \& VerWys, C. A. (2002). Repetition blindness, forward and backward. Journal of Experimental Psychology: Human Perception \& Performance, 28, 137-149.

Perfect, T. J., Moulin, C. J. A., Conway, M. A., \& Perry, E. (2002). Assessing the inhibitory account of retrieval-induced forgetting with implicit-memory tests. Journal of Experimental Psychology: Learning, Memory, \& Cognition, 28, 1111-1119.

Plaut, D. C., \& Booth, J. R. (2000). Individual and developmental differences in semantic priming: Empirical and computational support for a single-mechanism account of lexical processing. Psychological Review, 107, 786-823.

Pratt, J., Spalek, T. M., \& Bradshaw, F. (1999). The time to detect targets at inhibited and noninhibited locations: Preliminary evidence for attentional momentum. Journal of Experimental Psychology: Human Perception \& Performance, 25, 730-746.

RAtCLIFF, R, \& MCKoon, G. (1988). A retrieval theory of priming in memory. Psychological Review, 95, 385-408.

Rosch, E. H. (1978). Principles of categorization. In E. H. Rosch \& B. B. Lloyd (Eds.), Cognition and categorization (pp. 27-48). Hillsdale, NJ: Erlbaum.

RueCKL, J. G. (1990). Similarity effects in word and pseudoword repetition priming. Journal of Experimental Psychology: Learning, Memory, \& Cognition, 16, 374-391.

Sahakyan, L., \& Kelley, C. M. (2002). A contextual change account of directed forgetting. Journal of Experimental Psychology: Learning, Memory, \& Cognition, 18, 1064-1072.

Scarborough,D. L., Cortese, C., \& Scarborough, H. S. (1977). Frequency and repetition effects in lexical memory. Journal of Experimental Psychology: Human Perception \& Performance, 3, 1-17.

Seidenberg, M. S., Waters, G. S., Sanders, M., \& Langer, P. (1984). Pre- and postlexical loci of contextual effects on word recognition. Memory \& Cognition, 12, 315-328.

Stolz, J. A., \& Besner, D. (1999). On the myth of automatic semantic activation in reading. Current Directions in Psychological Science, $\mathbf{8}$, 61-65.

TIPPER, S. P. (1985). The negative priming effect: Inhibitory priming by ignored objects. Quarterly Journal of Experimental Psychology: Human Experimental Psychology, 37, 571-590.

Tipper, S. P., Meegan, D., \& Howard, L. A. (2002). Action-centered negative priming: Evidence for reactive inhibition. Visual Cognition, 9, 591-614.

Tulving, E. (1983). Elements of episodic memory. Oxford: Oxford University Press, Clarendon Press.

Tulving, E. (1995). Organization of memory: Quo vadis? In M. S. Gazzaniga (Ed.), The cognitive neurosciences (pp. 839-853). Cambridge, MA: MIT Press.

Vriezen, E. R., Moscovitch, M., \& Bellos, S. A. (1995). Priming effects in semantic classification tasks. Journal of Experimental Psychology: Learning, Memory, \& Cognition, 21, 933-946.

WhitTLESEA, B. W. A., \& JACOBY, L. L. (1990). Interaction of prime repetition with visual degradation: Is priming a retrieval phenomenon? Journal of Memory \& Language, 29, 546-565.

Whittlesea, B. W. A., \& Leboe, J. P. (2000). The heuristic basis of remembering and classification: Fluency, generation and resemblance. Journal of Experimental Psychology: General, 129, 84-106.

Woltz, D. J. (1996). Perceptual and conceptual priming in a semantic reprocessing task. Memory \& Cognition, 24, 429-440.

\section{NOTES}

1. Whittlesea and Jacoby (1990) used the term retrieval not in the sense of conscious recollection of the earlier event, but in the sense of selective access to an earlier experience, cued by the detail of a current experience.

2 . The same issues play out in longer term effects, such as directed forgetting and retrieval-induced forgetting. Whereas some investigators have taken these effects as demonstrations of the operation of inhibitory processes (e.g., M. C. Anderson, Bjork, \& Bjork, 1994; Bjork, 1989), others (e.g., Perfect, Moulin, Conway, \& Perry, 2002; Sahakyan \& Kelley, 2002) have argued that such an assumption is unnecessary.

(Manuscript received October 28, 2002; revision accepted for publication January 18, 2003.) 